\title{
A high sensitivity analog and digital position sensor for the detection of ferromagnetic target in automotive applications \\ Didier FRACHON ${ }^{1}$, \\ ${ }^{1}$ Moving Magnet Technologies SA - 1, rue Christiaan Huygens, 25000 Besançon, FRANCE \\ info@movingmagnet.com
}

\begin{abstract}
:
This paper introduces a new sensor technology of analog or digital position sensor for the detection of a ferromagnetic target in rotary or linear motion. This sensor is based on the use of a permanent magnet exhibiting a specific shape; this magnet is associated to a conical ferromagnetic part. The presented sensor has an improved sensitivity due to the use of this conical ferromagnetic part (acting as a flux concentrator) associated to a permanent magnet.
\end{abstract}

Key words: Digital and analog position sensor, Permanent magnet, Hall, Ferromagnetic target, Flux concentrator

\section{Introduction}

In the automotive world, the use of digital or analog position sensor for the detection of ferromagnetic target is quite well established. For this type of applications, there are mainly two types of sensor using different technologies:

- Inductive sensor using coil, a ferromagnetic core and a permanent magnet

- Sensor using permanent magnet associated to a magneto sensitive element (Hall sensor, GMR ...).

Amongst one of the well-known applications where one can find these kinds of sensor is the detection of the position of a ferromagnetic target installed on the camshaft of engines. One can see in figure 1 a target and a digital sensor.

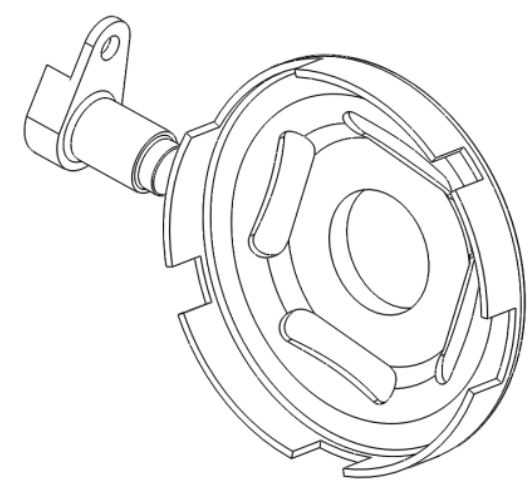

Fig. 1. Digital position sensor with a typical ferromagnetic target fitted to a camshaft.
A camshaft position sensor (for cylinder identification) is used to determine the engine cycle position, the information is then used by the ECU to provide individual cylinder ignition and injection control.

\section{Sensor using permanent magnet}

One of the advantages of sensors using permanent magnet as compared to inductive sensor is that they can easily exhibit TPO (True Power On) characteristic as you may have useful signal on the magneto-sensitive element (Hall sensor, GMR) without movement of the target to be detected.

MMT has already developed digital position sensor [1] such has the one shown below (see Fig. 2).

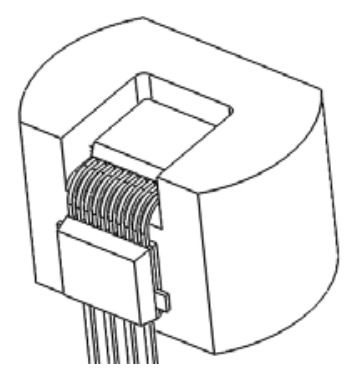

Fig. 2. Digital position sensor using a permanent magnet and Hall switch.

This sensor uses a permanent magnet and a basic and low cost digital Hall switch is integrated into a cavity created into the magnet.

In Fig. 3 below we give the flux density variation seen by the Hall switch as a function of the position of the target. 


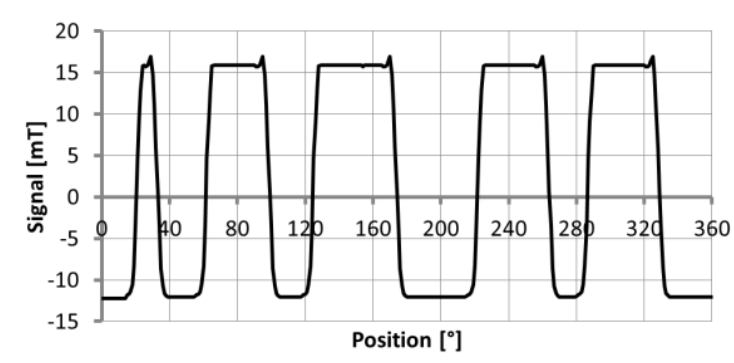

Fig. 3. Flux density variation as a function of the target position.

Using a Hall switch with a commutation threshold close to $0 \mathrm{mT}$, one gets the digital signal shown in Fig.4.

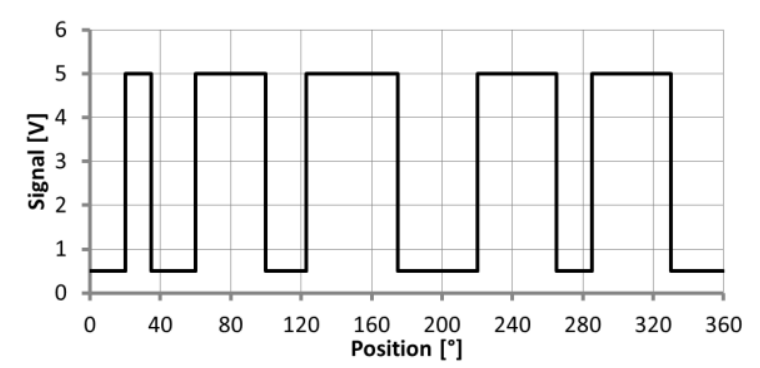

Fig. 4. Digital output signal of the sensor.

There are several advantages of using such structure. Amongst advantages, one can give:

- Using this specific shape for the magnet, one can design a sensor to work with low cost Hall switches working with a magnetic threshold close to $0 \mathrm{mT}$ leading to very low sensitivity to temperature,

- A higher field modulation, allows us to work with larger air gap and/or to get better signal to noise ratio. Indeed such embedded configuration allows us to add more magnetic material close to the ferromagnetic target that has to be detected and then to improve the performance of the sensor.

The magnetic induction generated by a magnetized cylindrical magnet with a hole in the center (see Fig.5) can be expressed by the following equation [2]:

$$
B(z)=\frac{B_{r}}{2 \mu_{r}}\left[\begin{array}{l}
\frac{z+L}{\sqrt{D^{2} / 4+(z+L)^{2}}}-\frac{z}{\sqrt{D^{2} / 4+z^{2}}}- \\
\frac{z+p}{\sqrt{d^{2} / 4+(z+p)^{2}}}+\frac{z}{\sqrt{d^{2} / 4+z^{2}}}
\end{array}\right]
$$

This equation is only valid along the axis of the magnet with parameters defined in the following figure:

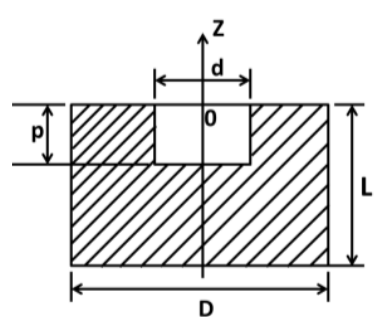

Fig. 5. Cylindrical magnet with a cylindrical hole.

- Where $z$ is equal to 0 on the top surface of the magnet. As the probe is "inside" the magnet, the magneto-sensitive is considered to be located at a $\mathrm{z}$ negative,

- $\mathrm{L}$ is the magnet thickness,

- $\mathrm{D}$ is the diameter of the magnet,

- $d$ is the diameter of the hole,

- $p$ is the depth of the hole.

One of the main parameters for this type of sensor is the magnetic field modulation that is defined by the difference of signal measured by the magneto-sensitive element with and without ferromagnetic target.

Thanks to the theory of magnetic image, it is possible to determine the field modulation as the function of the air gap $G$ between the sensor and the ferromagnetic target:

$$
\operatorname{Modulation}(z)=\frac{B_{r}}{2 \mu_{r}}\left[\begin{array}{l}
\frac{G-z+L}{\sqrt{D^{2} / 4+(z+L)^{2}}}-\frac{G-z}{\sqrt{D^{2} / 4+z^{2}}}- \\
\frac{G-z+p}{\sqrt{d^{2} / 4+(z+p)^{2}}}+\frac{G-z}{\sqrt{d^{2} / 4+z^{2}}}
\end{array}\right]
$$

The above equation is valid when considering a target much larger than the magnet. From this equation and if one considers $z<0$ one can easily notice that the field modulation increases as the $z$ position gets close to 0 . So the magneto-sensitive element needs to be as close as possible to the target and the depth of the hole needs to be as low as possible. The depth of the hole has to be chosen as close as possible to magneto-sensitive element thickness (see Fig.6).

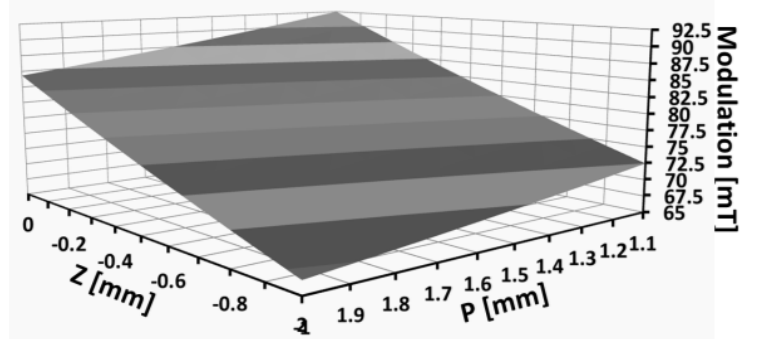

Fig. 6. Magnetic field modulation as a function of $z$ and $p$. 
If we now consider a cylindrical magnet without hole and a probe located on top of the magnet, the modulation is given by:

$$
\text { Modulation } \quad(z)=\frac{B_{r}}{2 \mu_{r}}\left[\frac{G-z+L}{\sqrt{D^{2} / 4+(z+L)^{2}}}-\frac{G-z}{\sqrt{D^{2} / 4+z^{2}}}\right]
$$

with z positive.

For a given magnet volume and for a given probe, we can plot the variation of the magnetic field modulation between the 2 configurations described above in order to show that configuration with the embedded magnetosensitive probe is much more attractive (see Fig.7):

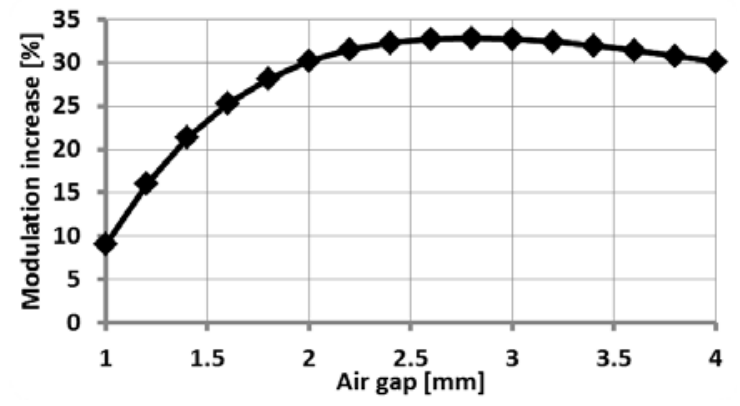

Fig. 7. Magnetic field modulation increase.

Indeed, on the performance point of view of digital position sensor, the higher the magnetic field modulation the better the capability to work at high mechanical air gap.

\section{Sensor with improved performance}

In order to be able to work at an even larger mechanical air gap we proposed an improved sensor using permanent magnet and a ferromagnetic flux concentrator [3].

We give in Fig. 8 below the basic structure of the improved sensor.

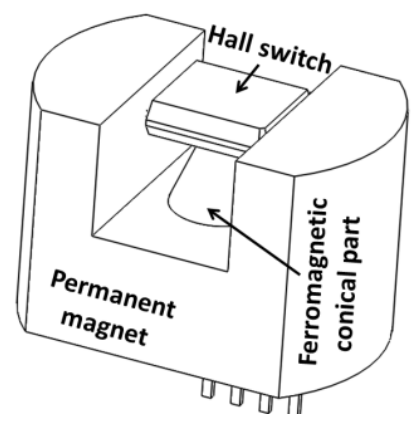

Fig. 8. Structure of the improved digital positions sensor.

The sensor is composed by 3 main components:

- $\mathrm{A} U$ shaped permanent magnet,
- A magneto-sensitive element (e.g. a Hall switch),

- A ferromagnetic conical part installed in a slotted magnet.

As one can see the structure is quite similar to the previously described sensor except the conical ferromagnetic part inserted in the magnet and below the magneto-sensitive element.

This additional part allows us to amplify the magnetic flux modulation.

In order to assess the improvement allowed by the new structure we simulated both sensors. One of the simulated structures is shown below in Fig.9 (reference sensor).
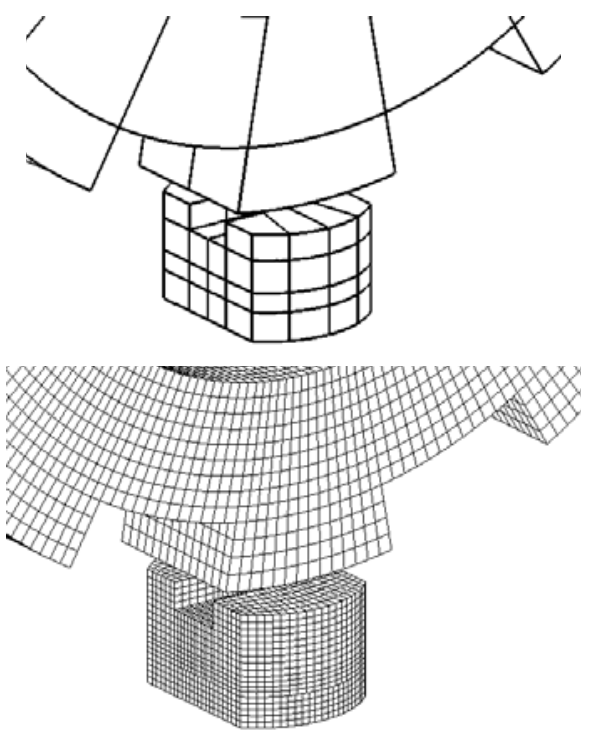

Fig. 9. Simulated structure of the reference sensor.

The variation of magnetic flux density measured by the magneto-sensitive element as a function of the angular position of the ferromagnetic target is given in Fig. 10 .

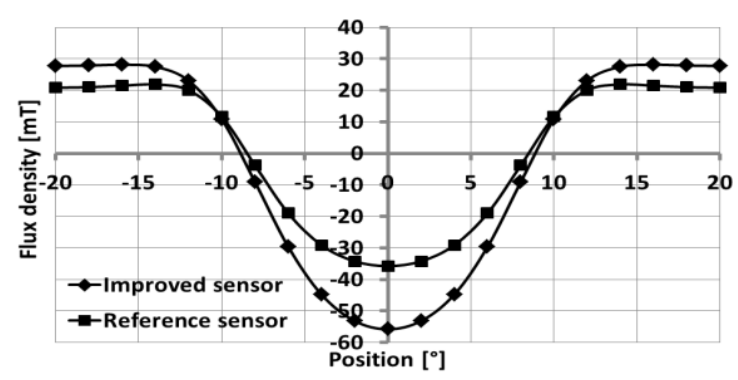

Fig. 10. Magnetic field modulation for the reference and improved sensors.

For ease of comparison, we used two sensors exhibiting the same volume and using of course the same permanent magnet material. One can easily notice the improvement that can be brought by this new structure. If we focus on the 
flux density modulation, we have an increase of about $50 \%$ of the flux density modulation. As already mentioned, this higher flux density modulation will allow us to work at larger air gap and/or to have a better signal to noise ratio.

\section{Mechanical robustness}

In this section, we are going to discuss about the sensitivity of the signal to the position of the additional conical ferromagnetic part.

We use the same structure as the one described in the previous section but for a different air gap.

We moved the conical ferromagnetic part along to directions perpendicular to the magnet axis as shown below:

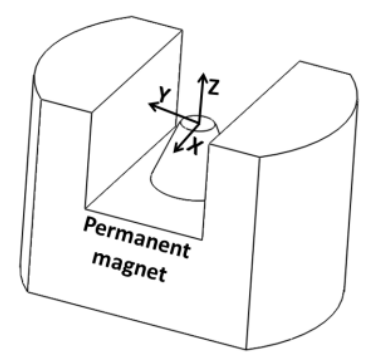

Fig. 11. Definition of the directions for the displacement of the ferromagnetic part.

We first give below the signal (see Fig. 12) seen by the Hall element as a function of the angular position of the target for 3 different configurations:

- Ferromagnetic conical part coaxial with the magnet

- Ferromagnetic conical part coaxial shifted along the $X$ axis $-0.1 \mathrm{~mm}$

- Ferromagnetic conical part coaxial shifted along the $Y$ axis $-0.1 \mathrm{~mm}$

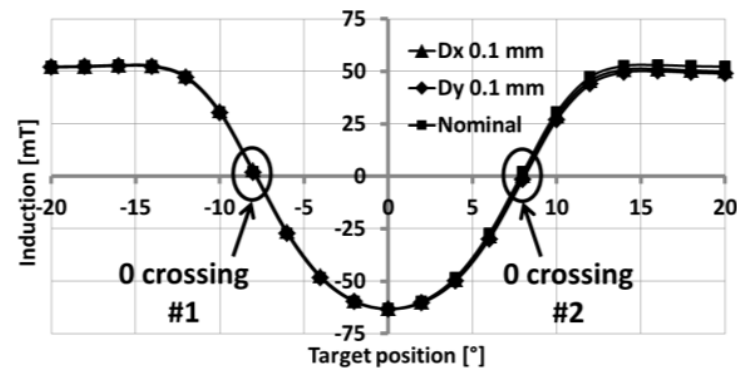

Fig. 12. Signal for the 3 predefined configurations.

As one can see from the above curves, the influence of a shift of the ferromagnetic part is quite low. As the sensor is supposed to deliver a change of signal at each 0 crossing, we plot below the variation of position of the 0 crossings for the 3 configurations above.

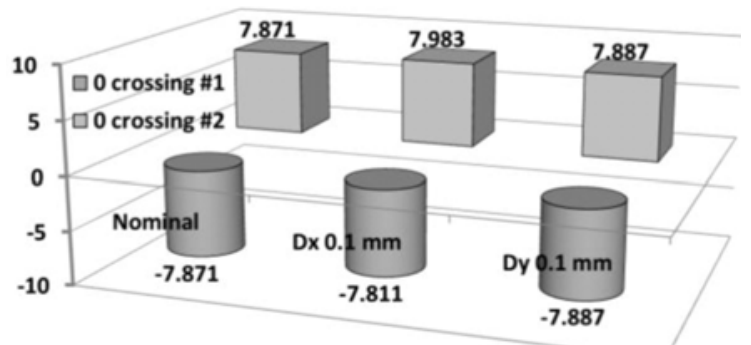

Fig. 13. Signal for the 3 predefined configurations.

The variation of the position of the 0 crossings is quite low as well. The shift along the $X$ axis is a bit more sensitive but it remains close to $0.1^{\circ}$ in the worst case.

\section{Dual output digital position sensor}

Using the improved structure of the digital position sensor, we have developed a new sensor type for applications requiring 2 outputs corresponding to 2 separate mechanical positions.

There are several challenges with this application:

- The sensor has to work with quite large magnetic airgap (4 to $6 \mathrm{~mm}$ ),

- $\quad$ The 2 digital outputs need to be shifted with one another as described below (see Fig.13).

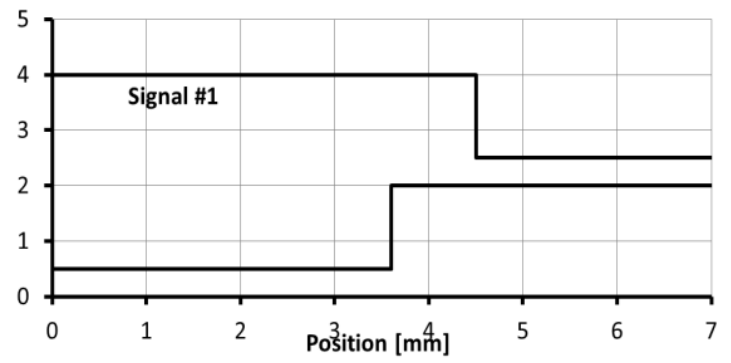

Fig. 14. Digital outputs of the sensor.

In this application, one has to detect the variation of airgap between the sensor and a ferromagnetic part. In the above Fig.14, the position is the distance between the top surface of the ferromagnetic target and the housing of the sensor. So the distance with the magnet is about 0.5 to $1 \mathrm{~mm}$ larger than the value shown in the graph above.

The sensor designed for this application needs "to measure" the distance between the sensor housing and a ferromagnetic target

In order to get 2 digital outputs, we use a dual output digital Hall switch. To get the desired "phase" shift between the 2 outputs, one has to design the magnet with a specific shape. 
Tab 1: Main parameters of the selected dual Hall switch

\begin{tabular}{|c|c|c|c|c|c|}
\hline \multirow{2}{*}{\begin{tabular}{|c|} 
Characteristic \\
MAGNETIC CHARACTERISTICS ${ }^{6}$
\end{tabular}} & Symbol & \multicolumn{4}{|c|}{ Min. Typ. Max. Unit' } \\
\hline & & & & & \\
\hline Operate Point: $B>B_{O P}$ & $B_{O P(A),} B_{O P(B)}$ & - & 7 & 30 & G \\
\hline Release Point $\mathrm{B}<\mathrm{B}_{\mathrm{RP}}$ & $B_{\text {RP }(A)} B_{\text {RP }(B)}$ & -30 & -7 & - & G \\
\hline 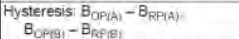 & $B_{\text {HYSSAY) }} B_{\text {HYS }}$ & 5 & 14 & 35 & $\mathrm{G}$ \\
\hline 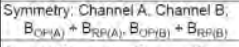 & $S Y M_{A-S Y M_{B}}$ & -35 & - & 35 & G \\
\hline Operate Symmetry: $B_{\mathrm{CP}(A)}-B_{\mathrm{OP} ; \mathrm{B})}$ & $S_{\text {SYE(OP) }}$ & -25 & - & 25 & G \\
\hline Release Symmetry. $B_{R P(A)}-B_{R P(B)}$ & $S Y M_{\text {AB:RPY }}$ & -25 & - & 25 & G \\
\hline
\end{tabular}

Simulations using Amperes [4] have been carried out in order to find the shape of the magnet allowing us to reach the desired $1 \mathrm{~mm}$ shift and to be compatible with mechanical tolerances.

We measured the sensor installed on the final application. Measurement results for 2 temperatures are shown in Fig.15 below. Measurements are given as a function of the angular position of the shaft holding the ferromagnetic target. At $25^{\circ} \mathrm{C}$, the average commutation positions for the signal \#1 and for signal \#2 are respectively equal to about $2.33^{\circ}$ and $2.9^{\circ}$.

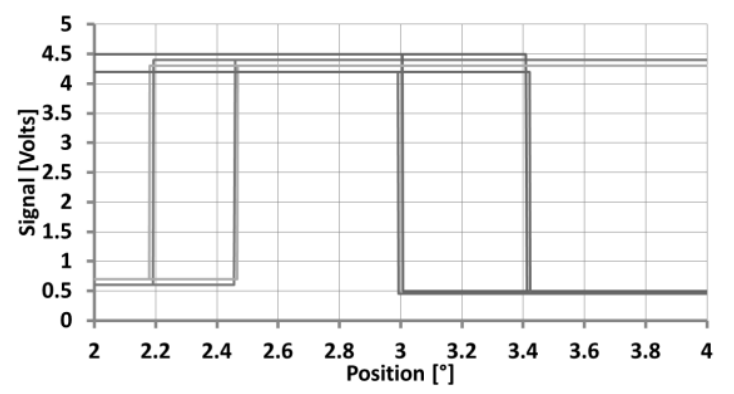

Fig. 15. Measured digital outputs of the sensor for 2 temperatures $\left(T=25^{\circ} \mathrm{C} \& T=85^{\circ} \mathrm{C}\right)$.

So knowing the distance between the shaft $(R=$ $88 \mathrm{~mm}$ ) and the center of the ferromagnetic target, one can convert the angular position of the shaft into an airgap between the sensor and the target. So the commutation occurs at about $3.58 \mathrm{~mm}$ for the signal \#1 about $4.45 \mathrm{~mm}$ for signal \#2, values that have to be compared respectively to the targeted values $3.6 \mathrm{~mm}$ and $4.5 \mathrm{~mm}$.

The measured prototype exhibits performances quite close to the requested one.

In Fig.15, we also show the sensor performance at $85^{\circ} \mathrm{C}$. As expected, the temperature has almost not effect on the output signals of the sensor. Thanks to a specific design, we are unsensitive to temperature because we can use a Hall switch with commutation thresholds close to $0 \mathrm{mT}$ (see Tab $1)$.

\section{Analog position sensor}

Using the same sensor structure as the one described in the above section, it is possible to get an analog position sensor. For such sensor, on has to use a linear programmable Hall sensor instead of using a digital Hall switch as previously mentioned.

We show below in Fig. 16 an example of analog position sensor to get the linear position of the ferromagnetic target. The target shown here is axisymmetrical, so the signal delivered by the sensor will be unsensitive to the angular position of the target.

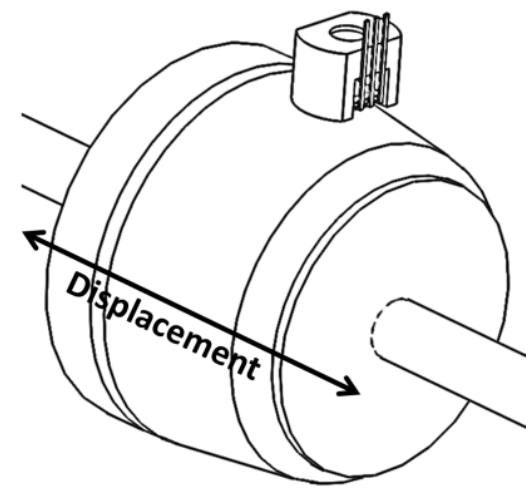

Fig. 16. Analog sensor with the ferromagnetic target for a linear position sensor.

In Fig.16 one can see:

- the sensor (magnet, conical ferromagnetic flux concentrator and linear Hall sensor),

- $\quad$ facing a ferromagnetic target exhibiting a specific profile.

As the target moves linearly, the airgap between the sensor and the target changes and so the signal measured by the Hall sensor. In order to get a linear signal as a function of the linear position of the target, one needs to find the correct profile of the target.

In Fig.17, we give the output signal delivered by the sensor shown above. As one can see the signal over the $17 \mathrm{~mm}$ stroke is quite linear and the linearity is better than $\pm 0.5 \%$ of the full scale.

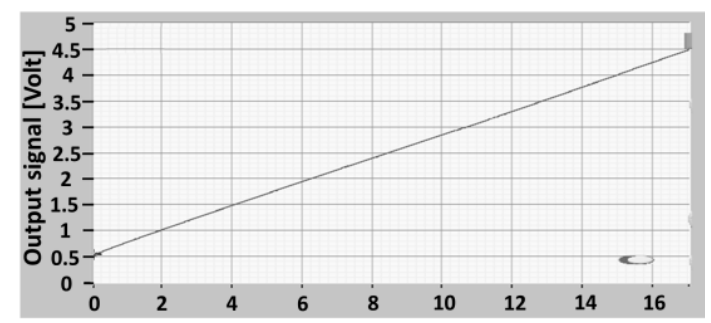

Fig. 17. Output signal of the analog linear position sensor of Fig. 16. 
To get such good performance, the sensor and the profile of the target need to be optimized as a system.

\section{Conclusion}

A digital Hall sensor with a specific design allowing a high stability over temperature range has been depicted. This structure is already in mass production for automotive camshaft sensor. A new high sensitivity digital and analog position sensor has been described as well. The new presented structure brings advantages in term of possible measurement air gap and size thanks to a much higher flux density modulation.

Indeed there is a strong trend to detect target motions through aluminum cases avoiding drilling holes to insert the sensor as closeas possible to the target. The targeted automotive applications are not only the highly dynamical camshaft sensor but as well quasi static applications (braking systems like pedal or oil piston for example).

[1] "Speed and/or position for detecting low and null speeds" - MMT patent EP0729582.

[2] "Magnétostatique" of E. DURAND by MASSON (1968)

[3] "linear or rotary position sensor with a permanent magnet for detecting a ferromagnetic target" MMT patent application WO2010034896.

[4] Boundary Element Method 3D simulation software - Amperes - Integrated Engineering Software

http://www.integratedsoft.com/Products/Amperes 